\title{
DIGITAL SUM INEQUALITIES AND APPROXIMATE CONVEXITY OF TAKAGI-TYPE FUNCTIONS
}

\author{
Pieter C. AllaArt
}

Abstract. For an integer $b \geqslant 2$, let $s_{b}(n)$ be the sum of the digits of the integer $n$ when written in base $b$, and let $S_{b}(N)=\sum_{n=0}^{N-1} s_{b}(n)$. Several inequalities are derived for $S_{b}(N)$. Some of the inequalities can be interpreted as comparing the average value of $s_{b}(n)$ over integer intervals of certain lengths to the average value of a beginning subinterval. Two of the main results are applied to derive a pair of "approximate convexity" inequalities for a sequence of Takagi-like functions. One of these inequalities was discovered recently via a different method by V. Lev; the other is new.

Mathematics subject classification (2010): Primary 11A63; secondary 26A27, 26A51.

Keywords and phrases: Digital sum, cumulative digital sum, Takagi function, approximate convexity.

\section{REFERENCES}

[1] P. C. AllaART, An inequality for sums of binary digits, with application to Takagi functions, J. Math. Anal. Appl. 381 (2011), no. 2, 689-694.

[2] Z. Boros, An inequality for the Takagi function, Math. Inequal. Appl. 11 (2008), no. 4, 757-765.

[3] H. DELAnge, Sur la fonction sommatoire de la fonction "somme des chiffres", Enseignement Math. 21 (1975), 31-47.

[4] M. Drmota, C. Mauduit And J. Rivat, The sum-of-digits function of polynomial sequences, J. Lond. Math. Soc. (2) 84 (2011), no. 1, 81-102.

[5] A. Házy And Zs. PÁLes, On approximately midconvex functions, Bull. London Math. Soc. 36 (2004), 339-350.

[6] V. F. LEV, Edge-isoperimetric problem for Cayley graphs and generalized Takagi function, preprint, arXiv:1202.2566 (2012)

[7] C. Mauduit And J. Rivat, Sur un probléme de Gelfond: la somme des chiffres des nombres premiers, Ann. Math. 171 (2010), no. 3, 1591-1646.

[8] C. Mauduit AND A. SÁRKÖZY, On the arithmetic structure of the integers whose sum of digits is fixed, Acta Arith. 81 (1997), no. 2, 145-173.

[9] M. D. MCIlroy, The number of 1's in binary integers: bounds and extremal properties, SIAM J. Comput. 3 (1974), no. 4, 255-261.

[10] K. B. STOLARSKY, Power and exponential sums of digital sums related to binomial coefficient parity, SIAM J. Appl. Math. 32 (1977), no. 4, 713-730.

[11] T. TAKagi, A simple example of the continuous function without derivative, Phys.-Math. Soc. Japan 1 (1903), 176-177, The Collected Papers of Teiji Takagi, S. Kuroda, Ed., Iwanami (1973), 5-6.

[12] J. R. Trollope, An explicit expression for binary digital sums, Math. Mag. 41 (1968), 21-25. 